\title{
Social and Spiritual Dimensions as Protective Factors in the Relationship between Acculturative Stress and Subjective Well-Being among International Students in Taiwan
}

\author{
Inna Reddy Edara \\ Graduate Institute of Educational Leadership and Development, Fu Jen Catholic University, Taiwan \\ Email:065049@mail.fju.edu.tw
}

How to cite this paper: Edara, I. R. (2018). Social and Spiritual Dimensions as Protective Factors in the Relationship between Acculturative Stress and Subjective WellBeing among International Students in Taiwan. Psychology, 9, 1582-1604. https://doi.org/10.4236/psych.2018.97096

Received: May 29, 2018

Accepted: July 2, 2018

Published: July 5, 2018

Copyright $\odot 2018$ by author and Scientific Research Publishing Inc. This work is licensed under the Creative Commons Attribution International License (CC BY 4.0).

http://creativecommons.org/licenses/by/4.0/

(c) (7) Open Access

\begin{abstract}
The purpose of this study was to use the resilience model in investigating the selected factors that reduce acculturative stress and promote well-being among international students in Taiwan. Research has indicated that acculturative stress has been identified as a major issue experienced by many international students in various host countries, resulting in an increase in mental illness and decrease in well-being. A few international studies suggested that factors such as social support and coping strategies mitigated the effects of acculturative stress on negative emotions. In the absence of such studies and as the international student population continues to increase in Taiwan, this project examined the role of social connectedness, social assurance, and spiritual well-being as potential mediators or protective factors in the relationship between acculturative stress and subjective well-being (positive affect and satisfaction with life) in a sample of international students. Data from 214 participants were subjected to regression analyses to analyze for mediation effects. The results indicated significant effects of social connectedness and spiritual well-being on subjective well-being. Of the two significant mediators, social connectedness emerged as a stronger mediator, accounting for $53 \%$ and $55 \%$ of the mediation effect on positive affect and satisfaction with life, respectively. Spiritual well-being accounted for $7 \%$ and $4 \%$ of the variance, respectively. These results validate the resilient and positive psychology model, in which the resilience part evades pathological signs when individuals are exposed to stressors, while the positive aspect promotes subjective well-being. These results are discussed in detail and the implication of such results for building appropriate social and spiritual resources and designing interventions are elucidated.
\end{abstract}




\section{Keywords}

Acculturative Stress, Positive Affect, Satisfaction with Life, Social

Connectedness, Social Assurance, Spiritual Well-Being

\section{Introduction}

Realizing the fact that international students are essential to colleges and universities in contributing to the cultural diversity of the student population, Taiwan has been designing programs to attract international students to pursue various educational and academic programs. Statistical summaries of overseas students in Taiwan (Ministry of Education, 2015) indicated that the total number of foreign students has increased from 25,023 in 2006 to 92,685 in 2014. Therefore, it will not be surprising to see that an increasing number of students will bring along an increase in challenges, both for the host country and international students. Research has indicated that although the majority of international students adapt reasonably well to the demands of the host culture and academic institutions, yet acculturative stress, which refers to stress induced by the adaptation process due to a change of cultural environment (Berry \& Ataca, 2000; Williams \& Berry, 1991), has been identified as a major issue experienced by many international students in various host countries (Zhang \& Dixon, 2003).

Studies from various countries suggested that acculturative stress leads to an increase in negative emotional states and a decrease in positive emotional well-being (Brown \& Holloway, 2007; Williams \& Berry, 1991). Some of the key factors may include loneliness, a mismatch of culture or cultural conflicts, lack of integration with host students, language barriers and communication difficulties, lack of social support, racial discrimination, financial stress and academic problems (see Furnham, 2004; Yen \& Stevens, 2004). There are also studies that involved the factors that mitigate the effects of acculturative stress on negative emotional states, such as social support and coping strategies (see Crockett, Iturbide, Stone, McGinley, \& Rafaelli, 2007; Lee \& Cifcti, 2014; Misra, Crist, \& Burant, 2003; Noh \& Kasper, 2003), sense of belongingness (Glass \& Westmont, 2014), and meaning in life (Glass \& Westmont, 2014; Pan, Wong, Chan, \& Joubert, 2008). A few studies conducted in Taiwan on international students' adaptation process and coping strategies also supported the general results (see Lin \& Su, 2011; Lu, 2011), but most of these studies in Taiwan were conducted as part of thesis, and thus were rather limited in their research scope with regard to both research subjects and analyses.

In addition, previous research on the psychopathological consequences of the acculturative stress has focused mainly on negative mental health aspects of depression and anxiety (see Noh \& Kasper, 2003; Rivera, 2007). However, recent focus on resilience model represents a significant research paradigm shift in the 
health-related fields. Resilience paradigm emphasizes a shift from a focus on negative risk factors and problems to a concentration on positive strengths, resources, and competencies of individuals (Pan, 2011). Hence, one of the approaches in applying the resilience paradigm to study acculturative stress and positive outcomes is to identify the significant protective factors and to investigate the mechanisms through which these factors work to reduce acculturative stress and promote well-being.

The purpose of this study, therefore, was to test the mediating effects of selective social and spiritual dimensions on the relationship between acculturative stress and subjective well-being among international students in Taiwan. First of all, while much of the previous work has examined the international students in the United States or other countries, there seems to be no evidence of such studies being done with international students in Taiwan. As indicated by the increasing number of international students in Taiwan and as acculturative stress has been proved again and again to be a normal experience of international students everywhere, it warrants an investigation of the acculturation process of international students in Taiwan in order to provide them with necessary tools for buffering stress. Second, as literature has indicated, because most of the previous studies focused on the effects of acculturative stress on negative emotional states, this study aimed to emphasize the positive outcomes by using the resilience framework of acculturation process. Third, this study also aimed to focus not only on the buffering effects in mitigating acculturative stress but also on the protective factors in promoting subjective well-being. Finally, none of the previous studies seemed to have employed spiritual variables in their research. This study, therefore, aimed to use spiritual dimensions along with social variables as mediating or protective factors in the relationship between acculturative stress and subjective well-being.

\section{Theoretical Underpinnings and Literature Review}

Given the emphasis of most of the previous studies on the relationship between acculturative stress and negative emotional states, and acknowledging the growing importance of positive psychology along with resilience framework in overall human functioning, this paper intended to base the research on positive psychology and resilience framework in buffering stress and promoting positive outcomes. The rationale for such an approach is explained in the following paragraphs.

\subsection{Resilience Framework}

Resilience is understood as the maintenance of positive adjustment under challenging stressful life conditions. Luthar, Cicchetti, and Becker (2000) referred to resilience as a dynamic process of positive adaptation within the context of some adversity. Luthar and colleagues evaluated that the thrust of resilience research has been a systematic search for protective forces that assist in the healthy adap- 
tation in the midst of various adverse conditions. Early efforts in resilience research primarily focused on the personal qualities of resilient people. However, as research in resilience evolved, various researchers increasingly acknowledged that resilience may derive from protective factors external to the people and they even began understanding the processes of how protective factors may contribute to positive outcomes. Protective factors are those variables or mechanisms that might modify the negative effects of adverse life circumstances on given outcomes in a positive direction (Luthar, Lyman, \& Crossman, 2014).

Further, recognizing the heterogeneity in adjustment levels across resilient domains, researchers now tend to use more specific terms to identify domains in which resilience is manifested; domains such as academic resilience, emotional resilience, social resilience, behavioral resilience (Luthar, Lyman et al., 2014) or psychological resilience (Ong, Bergeman, Bisconti, \& Wallace, 2006). Such an attempt to understand the underlying resilient mechanisms has been seen as essential for advancing theory as well as for designing appropriate intervention strategies.

\subsection{Resilience and Positive Psychology}

Luthar, Lyman et al. (2014) cited many commonalities between the resilience framework and positive psychology, and elucidated ways in which progress in each of these areas might collectively maximize the promotion of well-being among individuals and society. Positive psychology is the scientific study of strengths and virtues, well-being, and optimal functioning. The underlying assumption of positive psychology is that persons who carry even the weightiest psychological burdens care about much more in their lives than just the relief of their pain and suffering (Lopez \& Gallagher, 2009; Peterson \& Park, 2003; Seligman, 2002; Seligman \& Csikzentmihalyi, 2000). In brief, while resilience researchers consider the evasion of psychopathology when individuals are exposed to severe stressors, positive psychology is concerned with promotion of the positive aspects of adjustment and well-being.

Therefore, it is important to assess models of resilience that hypothesize additive, moderating, and mediating effects of contributing variables on outcomes. These models are important, not only to test buffering or protective factors, but also because they can serve as models of intervention. For example, additive models suggest that more resources, such as social support, can mitigate the negative effects of acculturative stress on mental health or well-being. Moderating models test for interaction effects in which a variable functions to alter the effect of adversity on the outcome. Moderating variables, such as personality or individual differences, are usually active in a person's life, but they alter the impact of adversity when it occurs, and as such some people react differently than others to the same event because of such individual differences. Mediating variables or protective factors help us to understand the acculturative process and its effects on the outcomes. 


\subsection{Acculturative Stress, Its Correlates and Effects}

Misra, Crist, and Burant (2003) identified a general stress model that includes three major conceptual domains of stressors, stress outcomes, and stress mediators. Under the umbrella of this stress model, cross-cultural psychologists have been employing the concept of psychological acculturation to refer to the changes in an individual and eventual outcomes that occur as a result of cultural encounters and acculturation process. Cultural maintenance and cultural participation are two strategies in the acculturation process. In cases where a serious conflict exists between these strategies, individuals may experience an acculturative stress (Berry, 1997), which is referred to the stress induced by the adaptation process due to a change of cultural environment (Berry, 1997; Berry \& Ataca, 2000). Acculturative stress can arise from incongruent cultural values and practices, language difficulties (Gil, Vega, \& Dimas, 1994), experiences of discrimination (Gil et al., 1994; Jung, Hecht, \& Wadsworth, 2007), negative stereotypes (Jung et al., 2007), and lack of social sense and cultural knowledge (Markus \& Kitayama, 2003).

Williams and Berry (1991) suggested that an experience of acculturative stress may lead to various negative emotional states, such as negative affect, anxiety, and depression. Recent research has indicated the effects of acculturative stress on various outcomes. For example, some studies in the United States indicated that acculturative stress was significantly correlated with loneliness and depression for the non-European students (Jung et al., 2007); with depression for the Chinese students (Wei, Heppner, Mallen, Ku, Liao, \& Wu, 2007), and with history of suicide attempt among an ethnically diverse group of students (Gomez, Miranda, \& Polanco, 2011). Revollo, Qureshi, Collazos, Valero, and Casas (2011) reported acculturative stress as a risk factor of depression and anxiety among the Latin American immigrants in Barcelona. In Taiwan, a few scattered studies investigated the international students' adaptation processes (see Lin \& Su, 2011; Lu, 2011), but none of them appear to have studied the acculturative stress and its effects on various outcomes.

Therefore, on the one hand, these various studies suggest that acculturative stress may lead to significant negative emotional states. But, on the other hand, Misra et al.'s (2003) stress model indicates that there are some stress mediators or protective factors between acculturative stress and outcomes, and thus warrants that including protective factors in an acculturation process is very important for advancing both research and practice.

\subsection{Acculturative Stress and Protective Factors}

Protective factors are those factors that, on the one hand, ameliorate or reduce the potentially negative effects of the risk factors on the outcomes, and on the other hand, they promote the positive outcomes. Research has identified a number of protective factors that buffer stress or facilitate positive outcomes in diverse adverse situations. At the international level, various protective factors 
have been studied. Most widely investigated variables were social support as a protective factor between acculturative stress and psychological adjustment (Crockett, Iturbide, Stone, McGinley, \& Raffaelli, 2007) and between life stress and reactions to stressors (Misra, Crist, \& Burant, 2003); coping as a protective factor between stress and psychological well-being (Crockett et al., 2007), and meaning in life as a protective factor between acculturation and outcomes of positive affect and satisfaction with life (Pan et al., 2008). Other protective factors that were reported in research were assertiveness (Lee \& Ciftci, 2014); stereotypes (López-Rodrígueza, Zagefkab, Navasa, \& Cuadrado, 2014); sense-making (Pan, 2011); social connectedness (Yoon, Lee, \& Goh, 2008; Zhang \& Goodson, 2011), and social interaction (Zhang \& Goodson, 2011). There is no indication of studies in Taiwan that used protective factors in investigating the effects of acculturative stress.

As evident from the literature review, although social support was widely used in acculturation studies, particularly in studies in the United States, it appears that most of the times the social support construct was measured in tangible terms. There is no indication in the literature of using spiritual dimensions as protective factors in the acculturation studies. Given these reasons, this study made an attempt to investigate the domains of social belongingness and spiritual well-being as protective factors in the relationship between acculturative stress and subjective well-being.

\subsection{Social Belongingness}

Heinz Kohut's (1984) self-psychology proposed the person's need for belongingness. Later clinical and social observations have shown that belongingness or lack of it has been very important in today's society (Lee \& Robins, 1995). Lee and Robins (p. 232), citing Kohut (1984), said that people seek to confirm a subjective sense of belongingness in order to avoid feelings of loneliness and isolation. Conversely, it can also be said that people seek a subjective sense of belongingness in order to promote their well-being. As said earlier, social support has been a popular construct in research studies, including in the acculturation studies. Lee and Robins (1995) clarified the distinction between social support and social belongingness in that social support focuses more on the lack of an appropriate social environment, whereas sense of belongingness focuses more on the strengths or deficiencies within the self in relation to the social environment. In this sense, belongingness would function as a higher order construct of human connectedness, which is consistent with Kohut's (1984) theory.

In their literature review on empirical support for belongingness, Lee and Robins (1995) said that there is an indirect empirical research to support the need for sense of belongingness in predicting various outcomes, such as social satisfaction, well-being, and loneliness. Lee and Robins also suggested the limitations and problems in the research so far with regard to adequately measuring the sense of belongingness, and thus developed a valid and reliable self-report 
measures that tap aspects of belongingness as proposed by Kohut's (1984) theory of self-psychology. Lee and Robins' (1995) research study developed the Social Connectedness and the Social Assurance scales as two aspects of belongingness, which were used as social protective factors in the current study.

Lee and Robins (1995) explained that social connectedness is related to one's opinion of self in relation to other people, with an emphasis on emotional distance or connectedness between the self and other people. It reflects the personal struggles of trying to maintain the sense of belongingness, expressed as the sense of being human among humans and feeling a pervasive sense of security. Hendrickson, Rosen, \& Aune (2011) said that identifying with those who may be perceived as different from oneself gives one a sense of social connectedness. The social assurance aspect of belongingness is related to one's reliance on other people, with a special focus on the need for reassurance from others in social situations to sustain the sense of belongingness (Lee \& Robins, 1995).

The lack of social connectedness was shown to be strongly related to acculturative distress (Yeh \& Inose, 2003) and it was said to play a large role in the psychological well-being of international students (Ward, Bochner, \& Furnham, 2001). Social connectedness acted as a moderator in Korean immigrants' subjective well-being (Yoon \& Lee, 2010), and it was a significant mediator of the volunteering and well-being relationship (Brown, Hoye, \& Nicholson, 2012).

\subsection{Spiritual Well-Being}

There seems to be a growing consensus among social scientists that the spirituality of humanity is an ontologically existent phenomenon and an essential dimension of human life (Moberg, 2002; Ortiz, Villereal, \& Engel, 2000). Jerry (2003) stated that all human beings possess a drive to spirituality in order to have a connection with a deeper source of wisdom. The relationship between spirituality and mental health has received increasing attention in recent decades (Sawatzky, Ratner, \& Chiu, 2005; Weaver, Pargament, Flannelly, \& Oppenheimer, 2006). Along with increasing interest in spirituality, many spirituality scales have been developed and several reviews of them have been conducted (see King \& Crowther, 2004; Meezenbroek, Garssen, van den Berg, van Dierendonck, Visser, \& Schaufeli, 2012). Recently, Meezenbroek et al. (2012) conducted a review of various spirituality questionnaires that measure spirituality as a universal human experience and concluded that the multidimensional Spiritual Well-Being Questionnaire (SWBQ) is the most promising of all the measures in studying well-being and distress.

Gomez and Fisher (2003) developed the SWBQ, comprising of personal, communal, environmental, and transcendental domains. Spiritual well-being is the affirmation of life with oneself, others, nature, and the transcendental other. Integrating these four domains together, spiritual well-being can be described as a state of reflecting positive feelings, thoughts, and behaviors of relationships with oneself, others, nature and the transcendent, that in turn provide the indi- 
vidual with a sense of wholeness, satisfaction, joy, positive attitudes, love, harmony, and purpose in life (Gomez \& Fisher, 2003: p. 1976). Meezenbroek et al. (2012) said that the SWBQ has no confusion with Subjective Well-Being as only one item appears to be related to it, namely the item "developing joy in life".

\subsection{Subjective Well-Being}

Many of the previous studies focused on the effects of acculturative stress on negative emotional states. Even the studies involving the factors that mitigate the effects of acculturative stress included mostly the negative emotional states. Therefore, what seems to be a necessary step in further research on acculturation is advancing the resilience-based models of acculturation with positive psychology outcomes, such as subjective well-being.

Subjective well-being (SWB) is an area of positive psychology that analyzes people's cognitive and affective evaluations of their lives (Diener, 2000; Diener, Oishi, \& Lucas, 2009). SWB is considered as a broad concept that includes experiencing high levels of positive emotions, low levels of negative moods, and high levels of life satisfaction (Diener, Suh, Lucas, \& Smith, 1999; Diener, Oishi et al., 2009). Emotional responses are studied through their pleasant and unpleasant affects, which are found to be somewhat independent and have different correlates (Diener, Suh et al., 1999). In this study, one affective component conceptualized as positive affect and one cognitive component conceptualized as life satisfaction were used to evaluate SWB. Briefly stated, positive affect reflects the extent to which an individual feels enthusiastic, active, and alert. Life satisfaction refers to the cognitive and judgmental process of SWB in assessing one's quality of life according to one's chosen criteria (Diener, 1994; Diener, Oishi et al., 2009).

Research has indicated that various indicators of social behavior and spirituality have consistently emerged as significant predictors of positive affect (Watson \& Naragon, 2009). Frequency of contact with relatives, involvement in social organizations, and overall level of social activity moderately correlated with positive affect (Myers \& Diener, 1995). Relationship harmony and social support were strongly associated with life satisfaction (Kwan, Bond, \& Singelis, 1997). Various studies also indicated that the indices of spirituality have been robustly associated with positive outcomes of well-being (see Diener, 2000; Ellison \& Fan, 2008; Greenfield, Vaillant, \& Marks, 2009; Hackney \& Sanders, 2003).

\subsection{Research Hypotheses}

Based on the existing gaps in the literature on the relationship between acculturative stress and subjective well-being (SWB) among international students in Taiwan, this study examined the direct relation between acculturative stress and SWB, as well as the effects of social and spiritual protective factors. Specifically, the following hypotheses were tested:

1) Intercorrelations 
a) There would be significant negative correlations between acculturative stress and the rest of the mediating and outcome study variables. For instance, there would be a significant negative correlation between acculturative stress and positive affect, suggesting that people who go through higher levels of stress caused by acculturation process experience lower levels of positive affect, and vice versa.

b) There would be significant positive correlations between the domains of social belongingness, spiritual well-being and subjective well-being. For example, significant positive correlation between the aspects of social belongingness and satisfaction with life would suggest that one's satisfaction with life would increase with better social belongingness conditions.

2) Mediational Analyses

a) After controlling for the demographics, there would be significant direct effects of acculturative stress, domains of social belongingness and spiritual well-being on SWB.

b) After controlling for the demographics, there would be significant partial mediational effects of domains of social belongingness and spiritual well-being on the relationship between acculturative stress and SWB. In other words, the effect of acculturative stress on SWB would be reduced significantly after entering the mediating variables in the regression equation, indicating the protective nature of social and spiritual dimensions in buffering acculturative stress and promoting SWB among international students in Taiwan.

\section{Method}

\subsection{Procedure and Participants}

Data of international students studying at various universities in Taiwan were collected through online as well as paper and pencil surveys. All the participants acknowledged or signed an electronic or hardcopy version of an informed consent form before proceeding with the survey. A souvenir worth of US\$6 was given to the participants who completed the survey questionnaire. The research design was approved by the Internal Review Board of the researcher's respective institution.

A total of 242 international students in Taiwan answered the survey. After excluding 26 cases who were missing values non-randomly, and two cases who didn't fulfill the inclusion criteria, the final sample resulted in 214 participants. Some randomly missing values on certain variables were replaced by the mean values on the respective variables, and a few outliers on some of the variables were replaced by changing the values to the next highest or lowest non-outlier value for that particular variable (Cohen, Cohen, West, \& Aiken, 2003).

As indicated in Table 1, the sample consisted of more female students (56\%) than male students (43.5\%). As expected in any student population, $94.4 \%$ were single and $4.6 \%$ were married. Most of the students were either Christian (28\%) or Catholic (26.2\%), have been living in Taiwan for less than one year (59.3\%), 
Table 1. Sample demographics.

\begin{tabular}{|c|c|c|c|}
\hline Variables & Categories & Number & Percentage \\
\hline \multirow{3}{*}{ Gender } & Male & 93 & $43.5 \%$ \\
\hline & Female & 120 & $56.0 \%$ \\
\hline & Missing & 1 & $0.5 \%$ \\
\hline \multirow{4}{*}{ Marital Status } & Single & 202 & $94.4 \%$ \\
\hline & Married & 10 & $4.6 \%$ \\
\hline & Separated & 1 & $0.5 \%$ \\
\hline & Missing & 1 & $0.5 \%$ \\
\hline \multirow{9}{*}{ Religious Affiliation } & Christian & 60 & $28.0 \%$ \\
\hline & Catholic & 56 & $26.2 \%$ \\
\hline & Muslim & 19 & $8.9 \%$ \\
\hline & Buddhist & 41 & $19.2 \%$ \\
\hline & Hindu & 2 & $0.9 \%$ \\
\hline & Atheist & 13 & $6.1 \%$ \\
\hline & Agnostic & 5 & $2.3 \%$ \\
\hline & Other & 15 & $7.0 \%$ \\
\hline & Missing & 3 & $1.4 \%$ \\
\hline \multirow{10}{*}{ Ethnicity } & African & 27 & $12.6 \%$ \\
\hline & Arab & 1 & $0.5 \%$ \\
\hline & Asian & 119 & $55.6 \%$ \\
\hline & Caribbean & 2 & $0.9 \%$ \\
\hline & Caucasian & 27 & $12.6 \%$ \\
\hline & Hispanic & 13 & $6.1 \%$ \\
\hline & Melanesian & 17 & $7.9 \%$ \\
\hline & Pacific Islander & 1 & $0.5 \%$ \\
\hline & Polynesian & 3 & $1.4 \%$ \\
\hline & Turkish & 1 & $0.5 \%$ \\
\hline \multirow{5}{*}{ Student Status } & Language & 35 & $16.4 \%$ \\
\hline & Bachelor & 89 & $41.6 \%$ \\
\hline & Masters & 60 & $28.0 \%$ \\
\hline & Doctoral & 15 & $7.0 \%$ \\
\hline & Missing & 15 & $7.0 \%$ \\
\hline \multirow{7}{*}{$\begin{array}{c}\text { Length of Stay in } \\
\text { Taiwan }\end{array}$} & Less than one year & 127 & $59.3 \%$ \\
\hline & One to two years & 33 & $15.4 \%$ \\
\hline & Two to three years & 24 & $11.2 \%$ \\
\hline & Three to four years & 11 & $5.1 \%$ \\
\hline & Four to five years & 10 & $4.7 \%$ \\
\hline & More than five years & 7 & $3.3 \%$ \\
\hline & Missing & 2 & $1.0 \%$ \\
\hline
\end{tabular}

$\mathrm{N}=214$.

and are pursuing a bachelor degree (41.6\%). The sample was distributed among various ethnic groups, with the largest group consisting of Asian (55.6\%), followed by Caucasian (12.6\%) and African (12.6\%). The largest Asian group comprised of 33 participants each from Malaysia and Indonesia, 14 from Vietnam, 8 each from Japan and South Korea, and the rest from other Asian countries.

\subsection{Measures}

Acculturative Stress Scale for International Students (ASSIS). Sandhu and Asrabadi (1994) developed the ASSIS scale, which consists of 36 items on 7 
subscales, including Perceived Discrimination (8 items), Homesickness (4 items), Perceived Hate/Rejection (5 items), Fear (4 items), Stress due to Change (3 items), Guilt (2 items) and Nonspecific (10 items). Items are measured on a 5-point Likert scale, ranging from 1 (strongly disagree) to 5 (strongly agree). A total score is the sum of all seven subscales, ranges from 36 to 180 , with higher scores representing greater acculturative stress. Sample items include "I am treated differently in social situations" and "I feel sad leaving my relatives behind." The reliability coefficients for the ASSIS ranged from .92 to .95 (Constantine, Okazaki, \& Utsey, 2004; He et al., 2012; Sandhu \& Asrabadi, 1994; Wei et al., 2007).

Social Connectedness Scale (SCS). Social connectedness scale, developed by Lee and Robbins (1995), measures one's emotional distance or connectedness between the self and other people. The scale consists of 8 items, uses a 6-point Likert scale $(1=$ strongly agree to $6=$ strongly disagree $)$, and has a potential range of 8 to 48 , with higher scores indicating a greater perceived sense of connectedness. While item content reflects a sense of social disconnectedness and detachment, the measure was named the Social Connectedness Scale, reflecting the inverse relationship between the item content and the direction of the rating system. Sample items include, "I feel disconnected with the people around me" or “I don't feel related to anyone” (Lee \& Robins, 1995). Reported scale reliability ranged from .91 to .93 (Hendrickson et al., 2011; Lee \& Robbins, 1995).

Social Assurance Scale (SAS). Consisting of 8 items, the SAS assess a general need for a sense of belongingness, measured on a 6-point Likert scale $(1=$ strongly disagree to $6=$ strongly agree). The total score has a range of 8 to 48 , with higher scores reflecting a sense of assurance or confidence in social situations. Sample items include, "My life is incomplete without a buddy beside me." Research reported scale reliability as .77 (Lee \& Robins, 1995).

Spiritual Well-Being Questionnaire (SWBQ). The SWBQ has a total of 20 positively worded items, rated on a 5-point Likert scale, ranging from 1 (very low) to 5 (very high). Examples of items include, "developing a sense of identity" or "developing a love for other people" or "developing connection with nature" and "developing a personal relationship with the Divine/God." The Cronbach's alpha was .92 for all items together (Gomez \& Fisher, 2003).

Positive Affect (PA). Positive affect was measured by using the 10-item Positive Affect subscale of PANAS scale (Watson, Clark, \& Tellegen, 1988). The PANAS was developed so that positive and negative factors would emerge as orthogonal dimensions (separate 10-item scales) rather than bipolar ends of the same scale. In this model, high PA is a state of high energy and concentration, whereas NA is a state of general distress. Watson, et al. suggested that because of the independence of their scales, these items are pure markers of NA and PA, and thus can be used separately. The scale ratings range from 1 (very slightly or not at all) to 5 (extremely), with higher score indicating higher PA. The alpha reliabilities for PA ranged from .86 to .90 . 
Satisfaction with Life (SWL). Satisfaction with life was assessed by the 5-item Satisfaction with Life Scale (Diener, Emmons, Larsen, \& Griffin, 1985; Pavot \& Diener, 1993), measured on a Likert scale ranging from 1 (strongly disagree) to 7 (strongly agree). The total score ranges from 5 to 35 , with 31 to 35 indicating extremely satisfied, 20 representing a neutral point, and 5 to 9 indicating extremely dissatisfied. Sample items include, "In most ways my life is close to my ideal" or "I am satisfied with my life." The SWLS has a reliability coefficient of .87 .

Demographics. A demographic form was included to capture essential demographic information, including age, gender, ethnicity, nationality, religious affiliation, current education status, occupation, marital status, and duration of stay in Taiwan.

\section{Results}

\subsection{Scale Reliabilities and Intercorrelations}

As indicated in Table 2, reliability coefficients for all the measures were in an acceptable range, with values ranging from .95 (acculturative stress) to .85 (satisfaction with life). This good internal consistency of the measures suggests that the items that make up the respective scale seem to measure rather accurately the intended underlying construct.

Assessing the correlational estimates as per Cohen's (1988) criteria (small $=.10$; medium $=.30$, and large $=$ above .50$)$ and as reported in Table 2, the significant correlations vary from .11 to .58 , except for the social assurance which does not have a significant correlation with the dimensions of subjective well-being. There is a high correlation between acculturative stress and social connectedness $(r=-.58, p<.01)$, suggesting that lack of social connectedness increases acculturative stress. There is also high correlation between spiritual well-being and positive affect $(r=.57, p<.01)$, indicating that strong sense of spirituality leads to greater positive feelings. The strong correlation between positive affect and satisfaction with life $(r=.37, p<.01)$ suggests that both the affective and cognitive dimensions together form subjective well-being.

\subsection{Mediation Analyses}

The most popular analytic strategy of testing mediator hypotheses is a straightforward application of multiple regression analysis (Baron \& Kenny, 1986; Frazier, Tix \& Baron, 2004). According to this strategy, dependent variable is regressed onto independent variable to show that there is a significant relation between them, followed by the regression of dependent variable simultaneously onto independent variable and mediator to show that the mediator is significantly associated with the dependent variable even when the independent variable is statistically controlled. Finally, the strength of the relationship between independent variable and dependent variable is investigated to see if it is significantly reduced when the mediator is added to the regression model (Baron \& 
Table 2. Reliability Coefficients ( $\alpha$ ), Means, Standard Deviations (SD), and Correlations.

\begin{tabular}{|c|c|c|c|c|c|c|c|c|c|}
\hline & $\alpha$ & Mean & $S D$ & 1 & 2 & 3 & 4 & 5 & 6 \\
\hline 1) Acculturative Stress & .95 & 85.28 & 20.09 & ---- & $-.11^{\star}$ & $-.58^{\star *}$ & $-.19^{* *}$ & $-.24^{\star *}$ & $-.18^{\star *}$ \\
\hline 2) Spiritual Well-Being & .88 & 75.75 & 11.25 & & --- & $.19^{* *}$ & $.21^{* *}$ & $.57^{\star *}$ & $.32^{* *}$ \\
\hline 3) Social Connectedness & .94 & 21.17 & 7.74 & & & ---- & $.15^{*}$ & $.33^{* *}$ & $.24^{* *}$ \\
\hline 4) Social Assurance & .86 & 28.75 & 7.65 & & & & ---- & .01 & .07 \\
\hline 5) Positive Affect & .88 & 35.43 & 6.67 & & & & & ---- & $.37^{* *}$ \\
\hline 6) Satisfaction with Life & .85 & 23.61 & 5.85 & & & & & & --- \\
\hline
\end{tabular}

$N=214 ; p^{* *}<.01 ; p^{*}<.05$

Kenny, 1986). Preacher \& Kelley (2011) proposed a formula to estimate the relative magnitude of mediation effect.

The results of mediation analyses with spiritual well-being, social connectedness and social assurance as three mediators between acculturative stress and positive affect are shown in Figure 1. As indicated in Figure 1, there is a significant mediation effect of spiritual well-being (Sobel $z=-1.61, p<.05 ; \Delta c=-.17$, $p<.01$ ) and social connectedness (Sobel $z=-4.57, p<.001 ; \Delta c=-.07, p<.05$ ) in the relationship between acculturative stress and positive affect. Using Preacher \& Kelly's (2011) formula, $a b / a b+\Delta c$, the mediation effect size is .27 and .73 , respectively. That is, $7 \%$ and $53 \%$ of variation in the relationship between acculturative stress and positive affect is accounted for by spiritual well-being and social connectedness, respectively. Social assurance didn't show a significant mediation effect in the relationship between acculturative stress and positive affect.

The results of mediation analyses with spiritual well-being, social connectedness and social assurance as three mediators between acculturative stress and satisfaction with life are shown in Figure 2. As indicated in Figure 2, there is a significant mediation effect of spiritual well-being (Sobel $z=-1.54, p<.05 ; \Delta c=$ $-.14, p<.05$ ) and social connectedness (Sobel $z=-4.57, p<.001 ; \Delta c=-.05, p$ $<.05)$ in the relationship between acculturative stress and satisfaction with life. Using Preacher \& Kelly's (2011) formula, the mediation effect size is .20 and .74, respectively. That is, $4 \%$ and $55 \%$ of variation in the relationship between acculturative stress and satisfaction with life is accounted for by the spiritual well-being and social connectedness, respectively. Social assurance didn't emerge as a significant mediator in the relationship between acculturative stress and satisfaction with life.

\section{Discussion}

Since various studies have indicated that stress resulting from acculturation and adjustment has been a major issue experienced by many international students in various host countries located in Europe and North America (Zhang \& Dixon, 2003), it would not be an exaggeration to say that the increasing number of 


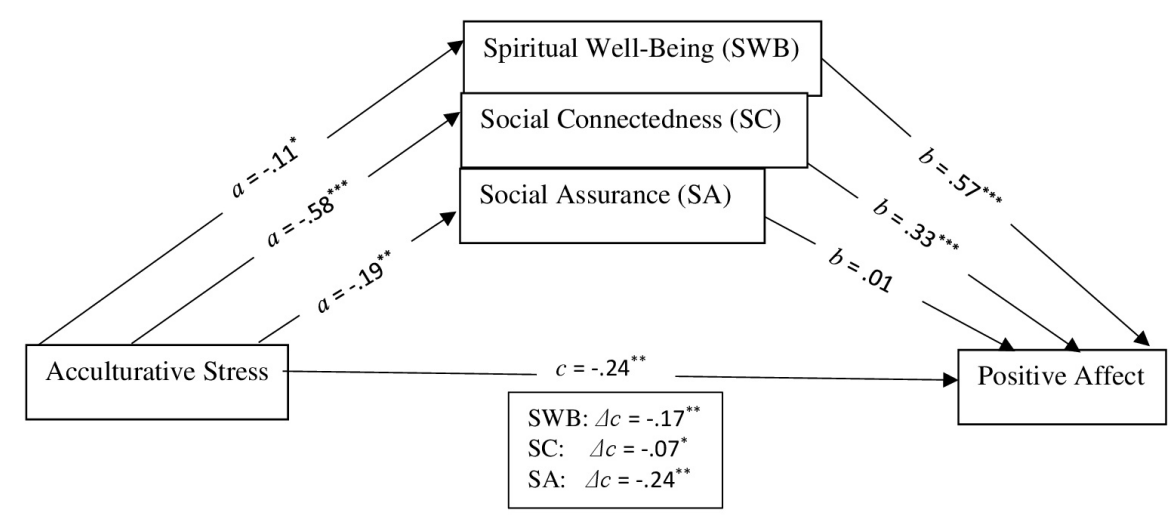

Figure 1. Mediation Effects of SWB, SC, and SA on the Relationship between Acculturative Stress and Positive Affect $N=214 ; p^{* * *}<.001, p^{* *}<.01 ; p^{*}<.05$. All the values are standardized coefficients.

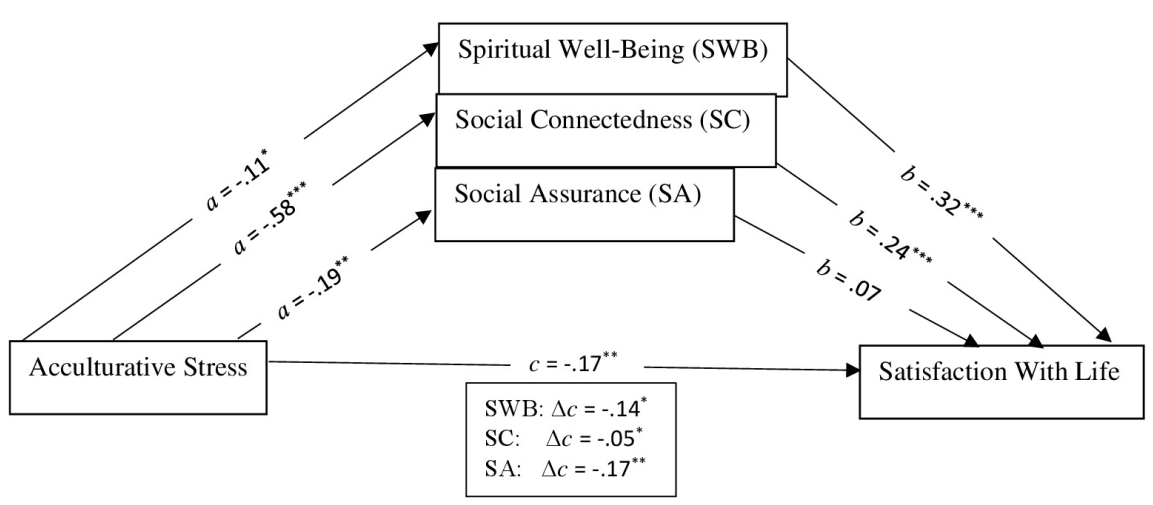

Figure 2. Mediation Effects of SWB, SC, and SA on the Relationship between Acculturative Stress and Satisfaction with Life. $N=214 ; p^{* * *}<.001, p^{* *}<.01 ; \mathrm{p}^{*}<.05$. All the values are standardized coefficients.

international students in Taiwan might also have to deal with stress that is both similar to the one experienced by students in other countries and specific to the situation of Taiwan. As measured by the Acculturative Stress Scale for International Students, with total scores ranging from 36 to 180, and higher scores representing greater acculturative stress (Sandhu \& Asrabadi, 1994), the sample in this study had a mean score of 85.28 , suggesting moderate levels of acculturative stress.

Using Misra et al.'s (2003) conceptual domains of stress process that includes stressors, stress mediators and stress outcomes, and as suggested by the significant results in this study and the contents of the items of the acculturative stress measurement, the international students in Taiwan experience moderate levels of acculturative stress through perceived discrimination and hatred, fear, homesickness, and some sort of culture shock. Berry (1997) said that cultural maintenance and cultural participation are two important strategies in the acculturation process of people living in all pluralistic societies. Acculturative stress is experienced when there is a serious conflict between these two strategies. For example, homesickness among the research participants, measured as part of the 
acculturative stress, indicates over-maintenance of home culture, whereas culture shock indicates the lack of participation in the host culture; thus leading to conflict between these two strategies and resulting in moderate levels of acculturative stress. Hence, as suggested by the significant correlational and regression mediational analyses, the outcomes of such levels of stress are decreased positive affect and satisfaction with life. As per Misra and his colleagues' (2003) stress model, what is necessary in dealing with such stress is conceptualizing and implementing stress mediators.

Therefore, in analyzing the ways that the international students in Taiwan might need to deal with their acculturative stress, this study employed the resilient model of mediational analyses with social and spiritual dimensions playing significant roles in mitigating the acculturative stress and promoting subjective well-being. Various researchers increasingly admit that resilience, as a dynamic process of positive adaptation within the context of some challenging and stressful life conditions, may not only be derived from protective factors external to the people undergoing stressful life conditions, but it may also contribute to positive outcomes (Luthar et al., 2014). As the significant results in this study have indicated, both social and spiritual mediating dimensions modify the effects of acculturative stress in a positive direction toward heightening the levels of subjective well-being dimensions of positive affect and satisfaction with life. These results, thus, validate the resilience and positive psychology model, wherein the resilience part of the mediators evades pathological signs when individuals are exposed to severe stressors, while the focus on positive psychology role of the mediators indicates the subsequent promotion of the positive adjustment and well-being.

In other words, both social and spiritual dimensions, as indicated by the significant mediational results of this study, act as significant protective factors, which on the one hand, ameliorate the acculturative stress, and on the other hand, promote positive affect and satisfaction with life. In this study, social dimension was measured by Social Belongingness (Lee \& Robins, 1995), consisting of Social Connectedness and Social Assurance. The significant mediational results have indicated that social assurance, which is described as one's reliance on other people (Lee \& Robins, 1995), has no significant effect. Whereas, social connectedness played a very significant mediating role in ameliorating acculturative stress and promoting subjective well-being. With mean value of 21.17 on a potential range of 8 to 48 , the participants' perceived sense of social connectedness was below average. Therefore, in line with Kohut's (1984) self-psychology, the participants of this study appeared to seek a subjective experience of belongingness and connectedness in order to avoid stress and promote well-being.

Unlike social support, which focuses more on the lack of an appropriate social environment, the sense of social connectedness focuses more on the strengths or deficiencies within the self in relation to the sociocultural environment (Lee \& Robins, 1995). This way of understanding appears to be consistent with the sig- 
nificant results of this study, wherein the subjects became aware of their strengths or deficiencies in their social connectedness, with deficiencies leading to more acculturative stress and strengths leading to more positive affect and satisfaction with life. In addition, as social connectedness is the internal process of self-awareness, so also both positive affect and satisfaction with life, the affective and cognitive components, respectively, are part of an individual's internal processes.

Spiritual dimension, as measured by Spiritual Well-Being (Gomez \& Fisher, 2003), also emerged as a significant protective and mediating factor in the relationship between acculturative stress and subjective well-being dimensions of positive affect and satisfaction with life. A mean score of 75.75 on a potential range of 20 to 100 suggests that the participants experienced a relatively high spiritual well-being. This result is rather consistent with the literature, which indicated that human spiritual dimension is an ontologically existent phenomenon, making it an essential dimension of human life, which drives people innately towards a healthy and meaningful life (see Jerry, 2003; Moberg, 2002; Ortiz et al., 2000). Although the mediating effect of spiritual well-being was moderate, the significant effect still underscores the importance of spiritual dimension as a protective factor in ameliorating acculturative stress and promoting subjective well-being dimensions of positive affect and satisfaction with life.

Given that the international students are bound to experience a conflict between maintaining one's own cultural values while participating in the cultural expectations of the host country, the personal, communal, environmental and transcendental factors of one's spiritual wellness (Gomez \& Fisher, 2003) do appear to play a moderate significant mediating and protective role in the lives of the participants. Like in the social connectedness, which is an internal process, spirituality also implies a clear sense of self-identity and deeply felt internal connection with other people, nature, and some form of transcendence. Such a sense of social, environmental, and spiritual connection leads to a state of reflecting positive feelings of affectivity and satisfaction (Gomez \& Fisher, 2003). Consequently, when people use their spiritual strength as a protective factor in the midst of stressful conditions, the potentially negative effects of acculturative stress risk factors on the outcomes of subjective well-being are ameliorated or substantially reduced.

\section{Implications and Future Directions}

The significant results of this study contribute to the understanding of international students' educational and cultural experiences in Taiwan context. The findings of the study yield significant information to assist educational institutions in providing necessary help to the international students in reducing their acculturative stress and increasing their subjective well-being by integrating appropriate social and spiritual resources and support systems in the student recruitment, academic planning, and institutional resource allocation. 
For example, although previous studies have indicated the role of various protective factors, such as personality traits and social support, the significant mediating role of social connectedness suggests that the participants seek a subjective experience of connectedness in order to avoid stress of living in a new cultural environment and promote their well-being. In comparison to the previous studies which used social support (Crockett et al., 2007), the social connectedness used in this research calls for focused interventions that draw on the strengths within the individuals in relation to their new sociocultural environment (Lee \& Robins, 1995).

Furthermore, on the basis of the resilience approach and positive psychology orientation, this research aimed to contribute to the literature by using social and spiritual dimensions as protective factors in buffering acculturative stress and enhancing subjective well-being among international students in Taiwan. It is expected that the significant results will help the educational institutions in Taiwan to design practical social and spiritual interventions in assisting their international students to experience a stress-free acculturation process, create a conducive academic environment, and promote their well-being. Moreover, as this study's significant results have indicated, an attempt to understand underlying resilient mechanisms is essential for designing appropriate psychosocial and spiritual intervention strategies in ameliorating stress and promoting positivity and well-being. Williams and Berry (1991) suggested that the experience of acculturative stress may lead to various negative emotional states, such as loneliness, negative affect, anxiety, depression, and even suicidal ideation and attempt. Therefore, while it is necessary to consider the opinions of the resilience researchers who work towards the evasion of psychopathology when individuals are exposed to severe stressors in unfamiliar environments, it is also imperative to focus on positive psychology in promoting the positive aspects of cultural adjustment and well-being.

This research study is not without limitations. One of the obvious limitations is that the majority of the study participants were from East Asia, whose cultural traditions and values, except for the language, are said to be closer to those of Taiwan. Such a proximity in cultural values and traditions for majority of the participants might have resulted in spurious effects, especially with regard to their scores on the acculturative stress. Secondly, as a consequence of the previous limitation, the overall significant results might not broadly generalize to the rest of the sample. Third, although the reliability coefficients of all the measures used in this study were very high, most of the measures seemed to have been developed in the Western countries with English as the primary language, and therefore, the possibility of misinterpretation of the items by the non-English speaking participants cannot be overlooked. Finally, it should be noted that the analyses in this study were correlational in nature, thus prohibiting causal relations.

Based on the implications and limitations, the future directions in this area of 
study should include larger samples, represented by more nationalities and ethnic backgrounds with largely differing cultural traditions and values in order to capture the accurate levels of acculturative stress or any other culture-related symptoms. Also, employing qualitative methods might shed more light on the subjective and phenomenological experiences of the participants. In addition, as indicated by Misra and his colleagues' (2003) three conceptual stress model, it should be a necessary step in researching the role of many more stress mediators or protective factors toward building models of stronger theories and more effective practices.

\section{Conclusion}

Based on the previous research studies in various countries that had indicated that acculturative stress was a major issue experienced by many international students in Europe and North America, it would not come as a surprise to know that the increasing number of international students in Taiwan might also have to deal with such stress. In fact, as suggested by the significant results in this study, the international students in Taiwan seem to experience moderate levels of acculturative stress through perceived discrimination and hatred, fear, homesickness, and some sort of culture shock. Obviously, the experience of the acculturative stress calls for appropriate ways of dealing with it. Therefore, in investigating the ways that the international students in Taiwan might need to deal with their acculturative stress, this study employed the resilient model of social and spiritual dimensions as playing significant mediating roles in mitigating the acculturative stress and promoting the subjective well-being. The analyses suggested that both social and spiritual mediating factors positively modify the effects of acculturative stress by elevating the levels of subjective well-being, and thus, validating the resilience and positive psychology model, wherein the resilience role of the mediators mitigates pathological effects and the focus on positive role of the mediators indicates the subsequent promotion of well-being. Finally, the significant results provide some useful information to the educational institutions in Taiwan in order to help the international students with the ways and resources in reducing their acculturative stress and increasing their subjective well-being.

\section{Funding}

This project was funded by the Office of Research and Development, Fu Jen Catholic University.

\section{References}

Baron, R. M., \& Kenny, D. A. (1986). The Moderator-Mediator Variable Distinction in Social Psychological Research: Conceptual, Strategic, and Statistical Considerations. Journal of Personality and Social Psychology, 51, 1173-1182.

https://doi.org/10.1037/0022-3514.51.6.1173

Berry, J. W. (1997). Immigration, Acculturation, and Adaptation. Applied Psychology: 
An International Review, 46, 5-34. https://doi.org/10.1111/j.1464-0597.1997.tb01087.x

Berry, J. W., \& Ataca, B. (2000). Cultural Factors. In G. Fink (Ed.), Encyclopedia of Stress (pp. 604-610). San Diego, CA: Academic Press.

Brown, K. M., Hoye, R., \& Nicholson, M. (2012). Self-Esteem, Self-Efficacy, and Social Connectedness as Mediators of the Relationship between Volunteering and Well-Being. Journal of Social Service Research, 38, 468-483. https://doi.org/10.1080/01488376.2012.687706

Brown, L., \& Holloway, I. (2007). The Initial Stage of the International Sojourn: Excitement or Culture Shock? British Journal of Guidance \& Counselling, 36, 33-49. https://doi.org/10.1080/03069880701715689

Cohen, J., Cohen, P., West, S. G., \& Aiken, L. S. (2003). Applied Multiple Regression/ Correlation Analysis for the Behavioral Sciences (3rd ed.). Mahwah, NJ: Lawrence Erlbaum.

Constantine, M. G., Okazaki, S., \& Utsey, S. O. (2004). Self-Concealment, Social Self-Efficacy, Acculturative Stress, and Depression in African, Asian, and Latin American International Students. American Journal of Orthopsychiatry, 74, 230-241. https://doi.org/10.1037/0002-9432.74.3.230

Crockett, L. J., Iturbide, M. I., Stone, R. A. S., McGinley, M., \& Rafaelli, M. (2007). Acculturative Stress, Social Support, and Coping: Relation to Psychological Adjustment among Mexican American College Students. Cultural Diversity and Ethnic Minority Psychology, 13, 347-355. https://doi.org/10.1037/1099-9809.13.4.347

Diener, E. (1994). Assessing Subjective Well-Being: Progress and Opportunities. Social Indicators Research, 31, 103-157. https://doi.org/10.1007/BF01207052

Diener, E. (2000). Subjective Well-Being: The Science of Happiness and a Proposal for a National Index. American Psychologist, 55, 34-43.

https://doi.org/10.1037/0003-066X.55.1.34

Diener, E., Emmons, R. A., Larsen, R. J., \& Griffin, S. (1985). The Satisfaction with Life Scale. Journal of Personality Assessment, 49, 71-75. https://doi.org/10.1207/s15327752jpa4901_13

Diener, E., Oishi, S., \& Lucas, R. E. (2009). Subjective Well-Being: The Science of Happiness and Life Satisfaction. In S. J. Lopez, \& C. R. Snyder (Eds.), Oxford Handbook of Positive Psychology (2nd ed., pp. 187-194). New York, NY: Oxford University Press. https://doi.org/10.1093/oxfordhb/9780195187243.013.0017

Diener, E., Suh, E. M., Lucas, R., \& Smith, H. (1999). Subjective Well-Being: Three Decades of Progress. Psychological Bulletin, 125, 276-302. https://doi.org/10.1037/0033-2909.125.2.276

Ellison, C. G., \& Fan, D. (2008). Daily Spiritual Experiences and Psychological Well-Being among US Adults. Social Indicators Research, 88, 247-271. https://doi.org/10.1007/s11205-007-9187-2

Frazier, P. A., Tix, A. P., \& Baron, K. E. (2004). Testing Moderator and Mediator Effects in Counseling Psychology Research. Journal of Counseling Psychology, 51, 115-134. https://doi.org/10.1037/0022-0167.51.1.115

Furnham, A. (2004). Foreign Students: Education and Culture Shock. The Psychologist, 17, 16-19.

Gil, A. G., Vega, W. A., \& Dimas, J. M. (1994). Acculturative Stress and Personal Adjustment among Hispanic Adolescent Boys. Journal of Community Psychology, 22, 43-54. https://doi.org/10.1002/1520-6629(199401)22:1<43::AID-JCOP2290220106>3.0.CO;2-T

Glass, C. R., \& Westmont, C. M. (2014). Comparative Effects of Belongingness on the 
Academic Success and Cross-Cultural Interactions of Domestic and International Students. International Journal of Intercultural Relations, 38, 106-119. https://doi.org/10.1016/j.ijintrel.2013.04.004

Gomez, J., Miranda, R., \& Polanco, L. (2011). Acculturative Stress, Perceived Discrimination, and Vulnerability to Suicide Attempts among Emerging Adults. Journal of Youth and Adolescence, 40, 1465-1476. https://doi.org/10.1007/s10964-011-9688-9

Gomez, R., \& Fisher, J. W. (2003). Domains of Spiritual Well-Being and Development and Validation of the Spiritual Well-Being Questionnaire. Personality and Individual Differences, 35, 1975-1991. https://doi.org/10.1016/S0191-8869(03)00045-X

Greenfield, E. A., Vaillant, G. E., \& Marks, N. F. (2009). Do Formal Religious Participation and Spiritual Perceptions Have Independent Linkages with Diverse Dimensions of Psychological Well-Being? Journal of Health and Social Behavior, 50, 196-212. https://doi.org/10.1177/002214650905000206

Hackney, C. H., \& Sanders, G. S. (2003). Religiosity and Mental Health: A Meta-Analysis of Recent Studies. Journal for the Scientific Study of Religion, 42, 43-55. https://doi.org/10.1111/1468-5906.t01-1-00160

He, F. X., Lopez, V., \& Leigh, M. C. (2012). Perceived Acculturative Stress and Sense of Coherence in Chinese Nursing Students in Australia. Nurse Education Today, 32, 345-350. https://doi.org/10.1016/j.nedt.2011.05.004

Hendrickson, B., Rosen, D., \& Aune, R. K. (2011). An Analysis of Friendship Networks, Social Connectedness, Homesickness, and Satisfaction Levels of International Students. International Journal of Intercultural Relations, 35, 281-295. https://doi.org/10.1016/j.ijintrel.2010.08.001

Jerry, P. A. (2003). Challenges in Transpersonal Diagnosis. The Journal of Transpersonal Psychology, 35, 43-59.

Jung, E., Hecht, M. L., \& Wadsworth, B. C. (2007). The Role of Identity in International Students' Psychological Well-Being in the United States: A Model of Depression Level, Identity Gaps, Discrimination, and Acculturation. International Journal of Intercultural Relations, 31, 605-624. https://doi.org/10.1016/j.ijintrel.2007.04.001

King, J. E., \& Crowther, M. R. (2004). The Measurement of Religiosity and Spirituality: Examples and Issues from Psychology. Journal of Organizational Change Management, 17, 83-101. https://doi.org/10.1108/09534810410511314

Kohut, H. (1984). How Does Analysis Cure? New York, NY: International Universities Press. https://doi.org/10.7208/chicago/9780226006147.001.0001

Kwan, V. S. Y., Bond, M. H., \& Singelis, T. M. (1997). Pancultural Explanations for Life Satisfaction: Adding Relationship Harmony to Self-Esteem. Journal of Personality and Social Psychology, 73, 1038-1051. https://doi.org/10.1037/0022-3514.73.5.1038

Lee, J.-Y., \& Ciftci, A. (2014). Asian International Students' Socio-Cultural Adaptation: Influence of Multicultural Personality, Assertiveness, Academic Self-Efficacy, and Social Support. International Journal of Intercultural Relations, 38, 97-105. https://doi.org/10.1016/j.ijintrel.2013.08.009

Lee, R. M., \& Robbins, S. B. (1995). Measuring Belongingness: The Social Connectedness and the Social Assurance Scales. Journal of Counseling Psychology, 42, 232-241. https://doi.org/10.1037/0022-0167.42.2.232

Lin, Y. H., \& Su, W. S. (2011). An Exploration of Leisure Transition and Adaptation of International Students in Taiwan. Leisure and Sports Journal, 10, 91-102.

Lopez, S. J., \& Gallagher, M. W. (2009). A Case for Positive Psychology. In S. J. Lopez, \& C. R. Snyder (Eds.), Oxford Handbook of Positive Psychology (2nd ed., pp. 3-6). New 
York, NY: Oxford University Press.

https://doi.org/10.1093/oxfordhb/9780195187243.001.0001

López-Rodrígueza, L., Zagefkab, H., Navasa, M., \& Cuadrado, I. (2014). Explaining Majority Members' Acculturation Preferences for Minority Members: A Mediation Model. International Journal of Intercultural Relations, 38, 36-46. https://doi.org/10.1016/j.ijintrel.2013.07.001

Lu, Q. X. (2011). A Study of Chinese Learning Difficulties and Coping Strategies for Foreign Students in Taiwan. Unpublished Thesis, Taipei: National Chengchi University.

Luthar, S. S., Cicchetti, D., \& Becker, B. (2000). The Construct of Resilience: A Critical Evaluation and Guidelines for Future Work. Child Development, 71, 543-562. https://doi.org/10.1111/1467-8624.00164

Luthar, S. S., Lyman, E., \& Crossman, E, J. (2014). Resilience and Positive Psychology. In M. Lewis, \& K. D. Rudolph (Eds.), Handbook of Developmental Psychopathology (pp. 125-140). New York, NY: Springer. https://doi.org/10.1007/978-1-4614-9608-3_7

Markus, H. R., \& Kitayama, S. (2003). Culture, Self, and the Reality of the Social. Psychological Inquiry, 14, 277-283. https://doi.org/10.1207/S15327965PLI1403\&4_17

Meezenbroek, E. D. J., Garssen, B., Berg, M. V. D., van Dierendonck, D., Visser, A., \& Schaufeli, W. B. (2012). Measuring Spirituality as a Universal Human Experience: A Review of Spirituality Questionnaires. Journal of Religion and Health, 51, 336-354. https://doi.org/10.1007/s10943-010-9376-1

Ministry of Education (2015). Non-Citizen Students in Universities, Colleges, and Junior Colleges.

http://english.moe.gov.tw/ct.asp?xItem $=16422 \& c t N o d e=11429 \& m p=1$

Misra, R., Crsit, M., \& Burant, C. J. (2003). Relationships among Life Stress, Social Support, Academic Stressors, and Reactions to Stressors of International Students in the United States. International Journal of Stress Management, 10, 137-157. https://doi.org/10.1037/1072-5245.10.2.137

Moberg, D. O. (2002). Assessing and Measuring Spirituality: Confronting Dilemmas of Universal and Particular Evaluative Criteria. Journal of Adult Development, 9, 47-60. https://doi.org/10.1023/A:1013877201375

Myers, D. G., \& Diener, E. (1995). Who Is Happy? Psychological Science, 6, 10-19. https://doi.org/10.1111/j.1467-9280.1995.tb00298.x

Noh, S., \& Kaspar, V. (2003). Perceived Discrimination and Depression: Moderating Effects of Coping, Acculturation, and Ethnic Support. American Journal of Public Health, 93, 232-238. https://doi.org/10.2105/AJPH.93.2.232

Ong, A. D., Bergeman, C. S., Bisconti, T. L., \& Wallace, K. A. (2006). Psychological Resilience, Positive Emotions, and Successful Adaptation to Stress in Later Life. Journal of Personality and Social Psychology, 91, 730-749. https://doi.org/10.1037/0022-3514.91.4.730

Ortiz, L., Villereal, S., \& Engel, M. (2000). Culture and Spirituality. Social Thought, 19, 21-26. https://doi.org/10.1300/J131v19n04_02

Pan, J. Y. (2011). A Resilience-Based and Meaning-Oriented Model of Acculturation: A Sample of Mainland Chinese Postgraduate Students in Hong Kong. International Journal of Intercultural Relations, 35, 592-603. https://doi.org/10.1016/j.ijintrel.2011.02.009

Pan, J. Y., Wong, D. F. K., Chan, C. L. W., \& Joubert, L. (2008). Meaning of Life as a Protective Factor of Positive Affect in Acculturation: A Resilience Framework and a Cross-Cultural Comparison. International Journal of Intercultural Relations, 32, 505-514. 
https://doi.org/10.1016/j.ijintrel.2008.08.002

Pavot, W., \& Diener, E. (1993). Review of the Satisfaction with Life Scale. Psychological Assessment, 5, 164-172. https://doi.org/10.1037/1040-3590.5.2.164

Peterson, C., \& Park, N. (2003). Positive Psychology as the Evenhanded Positive Psychologist Views It. Psychological Inquiry, 14, 143-147.

Preacher, K. J., \& Kelley, K. (2011). Effect Size Measures for Mediation Models: Quantitative Strategies for Communicating Indirect Effects. Psychological Methods, 16, 93-115. https://doi.org/10.1037/a0022658

Revollo, H. W., Qureshi, A., Collazos, F., Valero, S., \& Casas, M. (2011). Acculturative Stress as a Risk Factor of Depression and Anxiety in the Latin American Immigrant Population. International Review of Psychiatry, 23, 84-92. https://doi.org/10.3109/09540261.2010.545988

Rivera, F. I. (2007). Contextualizing the Experience of Young Latino Adults: Acculturation, Social Support and Depression. Journal of Immigrant and Minority Health, 9, 237-244. https://doi.org/10.1007/s10903-006-9034-6

Sandhu, D. S., \& Asrabadi, B. R. (1994). Development of an Acculturative Stress Scale for International Students: Preliminary Findings. Psychological Reports, 75, 435-448. https://doi.org/10.2466/pr0.1994.75.1.435

Sawatzky, R., Ratner, P. A., \& Chiu, L. (2005). A Meta-Analysis of the Relationship between Spirituality and Quality of Life. Social Indicators Research, 72, 153-188. https://doi.org/10.1007/s11205-004-5577-x

Seligman, M. E. P. (2002). Positive Psychology, Positive Prevention and Positive Therapy. In C. R. Snyder, \& S. J. Lopez (Eds.), Handbook of Positive Psychology (pp. 3-7). New York, NY: Oxford University Press.

Seligman, M. E. P., \& Csikszentmihalyi, M. (2000). Positive Psychology: An Introduction. American Psychologist, 55, 5-14. https://doi.org/10.1037/0003-066X.55.1.5

Ward, C., Bochner, S., \& Furnham, A. (2001). The Psychology of Culture Shock (2nd ed). Philadelphia, PA: Routledge.

Watson, D., \& Naragon, K. (2009). Positive Affectivity: The Disposition to Experience Positive Emotional States. In S. J. Lopez, \& C. R. Snyder (Eds.), Oxford Handbook of Positive Psychology (2nd ed., pp. 207-215). New York, NY: Oxford University Press.

Watson, D., Clark, L. A., \& Tellegen, A. (1988). Development and Validation of Brief Measures of Positive and Negative Affect: The PANAS Scales. Journal of Personality and Social Psychology, 54, 1063-1070. https://doi.org/10.1037/0022-3514.54.6.1063

Weaver, A. J., Pargament, K. I., Flannelly, K. J., \& Oppenheimer, J. E. (2006). Trends in the Scientific Study of Religion, Spirituality, and Health: 1965-2000. Journal of Religion and Health, 45, 208-214. https://doi.org/10.1007/s10943-006-9011-3

Wei, M., Heppner, P. P., Mallen, M. J., Ku, T. Y., Liao, Y. H., \& Wu, T. F. (2007). Acculturative Stress, Perfectionism, Years in the United States, and Depression among Chinese International Students. Journal of Counseling Psychology, 54, 385-394. https://doi.org/10.1037/0022-0167.54.4.385

Williams, C. L., \& Berry, J. W. (1991). Primary Prevention of Acculturative Stress among Refugees: Application of Psychological Theory and Practice. American Psychologist, 46, 632-641. https://doi.org/10.1037/0003-066X.46.6.632

Yeh, C. J., \& Inose, M. (2003). International Students' Reported English Fluency, Social Support Satisfaction, and Social Connectedness as Predictors of Acculturative Stress. Counseling Psychology Quarterly, 16, 15-28.

https://doi.org/10.1080/0951507031000114058 
Yen, W. J., \& Stevens, P. (2004). Taiwanese Students' Perspectives on Their Educational Experiences in the United States. International Education Journal, 5, 294-307.

Yoon, E., \& Lee, R. M. (2010). Importance of Social Connectedness as a Moderator in Korean Immigrants' Subjective Well-Being. Asian American Journal of Psychology, 1, 93-105. https://doi.org/10.1037/a0019964

Yoon, E., Lee, R. M., \& Goh, M. (2008). Acculturation, Social Connectedness, and Subjective Well-Being. Cultural Diversity and Ethnic Minority Psychology, 14, 246-255. https://doi.org/10.1037/1099-9809.14.3.246

Zhang, J., \& Goodson, P. (2011). Acculturation and Psychosocial Adjustment of Chinese International Students: Examining Mediation and Moderation Effects. International Journal of Intercultural Relations, 35, 614-627. https://doi.org/10.1016/j.ijintrel.2010.11.004

Zhang, N., \& Dixon, D. N. (2003). Acculturation and Attitudes of Asian International Students toward Seeking Psychological Help. Journal of Multicultural Counselling and Development, 31, 205-222. https://doi.org/10.1002/j.2161-1912.2003.tb00544.x 